\title{
Auf dem Weg zur Viszeralmedizin
}

\author{
Reinhard Bittner ${ }^{a}$ Wolfgang Kruis ${ }^{b}$ \\ a Klinik für Allgemein- und Visceralchirurgie, Marienhospital Stuttgart, \\ ${ }^{b}$ Evangelisches Krankenhaus Köln-Kalk, Deutschland
}

Das Gesundheitswesen befindet sich in ein einem nie zuvor da gewesenem Umbruch, der auch nicht vor den traditionellen Wurzeln des Selbstverständnisses der Ärzteschaft haltmacht. Im Besonderen sind es ökonomische Zwänge, die mehr und mehr in den Vordergrund rücken. Fragen nach Kosteneffektivität und Kosteneffizienz einer Behandlungsmethode beschäftigen zunehmend Kliniker, Verwaltungen und Politiker. Die Vermeidung von Doppeluntersuchungen und unnötigen Wartezeiten sowie die Entwicklung gemeinsamer Behandlungspfade und Leitlinien erfordern eine eng verzahnte interdisziplinäre Zusammenarbeit. Dies gilt im ganz besonderem Maß für die Viszeralchirurgie und die Gastroenterologie. Beide Fachdisziplinen behandeln die gleichen Patienten mit den gleichen Krankheitsbildern. Die nahtlose Abstimmung konservativer und operativer Maßnahmen ist nicht nur eine Conditio sine qua non für eine optimale Behandlung des Patienten, sondern auch von größtem ökonomischen Interesse. Gastroenterologie und Viszeralchirurgie müssen sich zur Viszeralmedizin verzahnen. Dies gilt nicht nur für den klinischen Alltag, sondern auch für die bei- den wissenschaftlichen Fachgesellschaften, die DGVS und die DGVC. Mit der gemeinsamen Jahrestagung 2005 in Köln wurde ein bedeutsames Signal für zukünftige Entwicklungen gesetzt. Aus einer Vielzahl hochkarätiger Vorträge wurden von uns einige, die exemplarisch die Interdisziplinarität herausstellen, zur Publikation in dem vorliegendem Sonderheft der Chirurgische GastroenteROLOGIE INTERDISZIPLINÄR ausgewählt. Die Manuskripte, alle von ausgewiesenen Experten geschrieben, beschreiben nicht nur den aktuellen wissenschaftlichen Stand, sondern sollen auch eine Art Leitfaden darstellen für die interdisziplinäre Diskussion in der täglichen Routine. Nur durch die Darstellung der Ergebnisse wissenschaftlicher Untersuchungen sowie evidenzbasierter Daten können unnötige Abstimmungsprobleme vermieden und effiziente interdisziplinäre Behandlungskonzepte realisiert werden.

Wir hoffen, mit dem gemeinsamen Kongress in Köln sowie dem daraus resultierendem Supplement einen Prozess in Gang gesetzt zu haben, der Vorbildcharakter hat und als ein Meilenstein auf dem Weg zur Viszeralmedizin bezeichnet werden kann.
Prof. Dr. Dr. h.c. Reinhard Bittner 